\title{
Parents attitudes toward the human papilloma virus (HPV) vaccine: A new concept in the State of Qatar
}

\author{
Mohamed A. Hendaus ${ }^{1,2,3}$, Manasik Hassan ${ }^{2,3}$, Moza Alsulaiti ${ }^{4}$, \\ Tasabeh Mohamed ${ }^{4}$, Reem Mohamed ${ }^{4}$, Dure Yasrab ${ }^{4}$, Hadeel Mahjoob ${ }^{4}$, \\ Ahmed H. Alhammadi ${ }^{1,2,3}$
}

${ }^{1}$ Department of Pediatrics, Section of Academic General Pediatrics, Sidra Medicine, Doha, ${ }^{2}$ Department of Clinical Pediatrics, Weill- Cornell Medicine, Doha, ${ }^{3}$ Department of Pediatrics, Hamad Medical Corporation, Doha, ${ }^{4}$ Depatment of Medical Education, Pediatric Residency Program, Hamad Medical Corporation, Doha, Qatar

\begin{abstract}
Background: Human papillomavirus (HPV) is one of the leading causes of cervical and genital cancer in both genders. Purpose: To delineate parental attitude regarding HPV in Qatar. Methods: A cross-sectional study using a questionnaire was conducted at Sidra Medicine, Qatar. Results: A total of 334 questioners were completed. More than $60 \%$ of the parents were not aware that HPV can cause cervical and genital cancer. When asked about the level of comfort in giving their children a vaccine that would prevent them from getting genital cancer, $77 \%$ of the participants answered "very comfortable." Interestingly, less than $4 \%$ of the parents stated that their children's primary care physicians ever mentioned that such a vaccine exists. When asked about the most preferable mode of receiving information regarding the HPV vaccine, 54\% preferred the clinician's office, followed by $34 \%$ of whom preferred social media. In terms of the preferred age to receive the vaccine, $45 \%$ of the participants preferred to administer the vaccine to their children before they were mature enough to understand sexual relations, while $22 \%$ recommended vaccination right before marriage and $15 \%$ preferred to wait till they were grown up and decide for themselves. Furthermore, only $42 \%$ of the caregivers agreed that it is important to explain to their children that the vaccine can protect against some of the sexually transmitted infections. Finally, approximately 20\% of the participants were not convinced about the HPV vaccine. Conclusion: A large proportion of parents residing in Qatar have a positive perception regarding the HPV vaccine. TheParents' attitudes and perceptions are considered indispensable targets for community health intervention. We will share the result of our study with the ministry of public health in Qatar with a goal to incorporate the HPV vaccine in the National Immunization Schedule.
\end{abstract}

Keywords: Children, HPV, pediatric, Qatar, vaccine

\section{Introduction}

Address for correspondence: Dr. Manasik Hassan, Department of Pediatrics, Hamad Medical Corporation, Doha - 26999, Qatar. E-mail: Mhassan17@hamad.qa

Received: 08-06-2020

Revised: 05-09-2020

Accepted: 08-09-2020

\begin{tabular}{|l|l|}
\multicolumn{2}{c}{ Access this article online } \\
\hline Quick Response Code: & \\
& Website: \\
& www.jmpc.com \\
& \\
\end{tabular}

Human papillomavirus (HPV) is transmitted mainly via sexual encounters. Two HPV types (16 and 18) cause $70 \%$ of cervical cancers and pre-cancerous cervical lesions. Moreover, HPV can cause cancers of the penis, vagina, anus, vulva, and oropharynx. Globally, the incidence of cervical cancer is around 5,70,000

This is an open access journal, and articles are distributed under the terms of the Creative Commons Attribution-NonCommercial-ShareAlike 4.0 License, which allows others to remix, tweak, and build upon the work non-commercially, as long as appropriate credit is given and the new creations are licensed under the identical terms.

For reprints contact: WKHLRPMedknow_reprints@wolterskluwer.com

How to cite this article: Hendaus MA, Hassan M, Alsulaiti M, Mohamed T, Mohamed R, Yasrab D, et al. Parents attitudes toward the human papilloma virus (HPV) vaccine: A new concept in the State of Qatar. J Family Med Prim Care 2021;10:2488-93. 
new cases and in 2018, an estimation of 3,11,000 women died from cervical cancer. ${ }^{[1,2]}$

The State of Qatar is an independent emirate in the Gulf Region. It is a peninsula, bordered by Saudi Arabia, and shares maritime borders with Bahrain, Iran, and the United Arab Emirates.

The country covers an area of $11,586 \mathrm{~km}^{2}\left(4,473 \mathrm{mi}^{2}\right)$ and has a population of 2.8 million inhabitants. ${ }^{[3]}$ In 2018, there were 19 new cervical cancer cases in Qatar. This type of cancer ranks as the fifth leading cause of female cancer and is the third most common female cancer aged 15-44 years in Qatar. ${ }^{[4]}$

The accessibility of the efficient and safe prophylactic HPV vaccine has opened up a new landscape for decreasing the cervical cancer issue, provided, the vaccines can be accessible through a national program. ${ }^{[5]}$ In 2006, the World Health Organization (WHO) introduced the HPV vaccine, but all the countries did not embed it into their immunization schedule. In 2017, more than 70 nations globally introduced the HPV vaccine into their routine vaccination schedule. ${ }^{[2]}$ The Center for Disease Control and Prevention (CDC) lists the HPV vaccine as safe and efficient. It is recommended to be administered to children 11 or 12 years of age. A catch-up schedule is recommended for boys and men through age 21 years and for girls and women through age 26 years if they have missed the opportunity to be vaccinated in the early teen years. ${ }^{[6-8]}$

The cultural satisfactoriness of a novice vaccine is a key pivotal element to be taken into consideration before introducing the vaccine to the public health system..$^{[9]}$

The HPV vaccine is minimally known in Qatar. Investigating parental attributes and perception of the HPV vaccine might assist in introducing the vaccine into our community. No studies regarding the HPV vaccine have been conducted in Qatar. Investigating parental perception and acceptability of the vaccine will be the best initial step in introducing the vaccine. The long-term plan and vision will be cervical cancer-free Qatar through vaccination, preventive medicine, and awareness.

\section{Materials and Methods}

\section{Study type}

A cross-sectional prospective study. The questions and the fact sheet have been adopted from different references, ${ }^{[4,7-16]}$ and modified to meet our cultural sensitivity. The experts reviewed the questions on the topic.

\section{Study location}

The study was conducted in the pediatric inpatient and outpatient departments of Sidra Medicine, the only tertiary institution in the State of Qatar at the time of the study.

\section{Sample size}

There are no identical studies in Qatar so our option was to extrapolate the sample size from one study ${ }^{[14]}$ conducted in the Arab world.

Sample Size for Frequency in a Population

Population size (for finite population correction factor or fpc) (N): 1000000

Hypothesized \% frequency of outcome factor in the population (p): 50\%+/-6

Confidence limits as $\%$ of 100 (absolute +/- \%) (d): 6\%

Design effect (for cluster surveys-DEFF): 1

Sample size (n) for various confidence levels

Confidence

Level (\%) Sample size

$95 \% 267$

$80 \% 115$

$90 \% 188$

$97 \% 327$

$99 \% 461$

$99.9 \% 752$

$99.99 \% 1051$

Equation

Sample size $(n)=\left[\operatorname{DEFF}^{*} N p(1-\mathrm{p})\right] /\left[\left(\mathrm{d} 2 / \mathrm{Z} 21-\alpha / 2 *(\mathrm{~N}-1)+\mathrm{p}^{*}(1-\mathrm{p})\right]\right.$

However, to account for many subgroup analyses and several secondary outcome measures, the plan is to enroll a total of 330 participants into this current research study.

\section{Study population}

Parents of children 9-18 years of age were approached while visiting the outpatient department for well visits or sick visits. In addition, parents of hospitalized children were also approached. A verbal consent form was provided before administering the questionnaire, and the participants were informed of why the information was being collected and how it would be used. Parents were informed that their participation was voluntary and we confirmed that their answers were anonymous and confidential. Participants did not receive any monetary or non-monetary compensation for participating in the study. In addition, we informed the participants that the project 
had received approval from Sidra Research Office and Sidra Institutional Board Review Committee (\#IRB 2019-0003).

\section{Questionnaire}

Parents were offered questionnaires in both Arabic and English languages. The self-administered questionnaire comprised of 20 items as follows:

a. Parent and children demographics (7 questions)

b. Knowledge and awareness of vaccines and genital cancer (4 questions)

c. Specific questions about HPV vaccine after reading the provided HPV fact sheet (8 questions)

d. Open-ended question for parental comments (1 question)

\section{Study period}

April 1, 2019 to March 30, 2020

\section{Statistical Analysis}

SPSS, version 21.0 (IBM corporation, Armonk, NY) was used for data analysis, and a two-sided $P$ value $<0.05$ was considered statistically significant. Qualitative and quantitative data measurements were exhibited as percentages. Descriptive statistics explained the demographics and other characteristics of parents and children. Associations between two or more categorical variables were appraised using the Chi-square test. For small cell frequencies, the Chi-square test with continuity correction factor or Fisher's exact test was used. Univariate and multivariate logistic regression analysis was used to assess the associations of various potential predictors and covariates. Missing data were not accounted for in the analysis.

\section{Results}

Total 334 questioners were completed (response rate $=94 \%$ ). More than $70 \%$ of the surveyed parents were between the ages of 20 and 39 years with the majority (75\%) being females, and 55\% were college graduates. Table 1 summarizes the demographic characteristics of the participants.

More than $60 \%$ of the parents were not aware that HPV can cause cervical and genital cancer. When asked about the level of comfort in giving their children a vaccine that would prevent them from getting genital cancer, $77 \%$ of the participants answered "very comfortable."

At this stage, the participants were given a fact sheet that explained in lay language about genital cancer and vaccination against HPV.

Interestingly, less than $4 \%$ of parents stated that their children's primary care physicians ever mentioned that such a vaccine exists. When asked about the most preferable mode of receiving information regarding the HPV vaccine, 54\% preferred the clinician's office, followed by $34 \%$ of whom preferred social
Table 1: Demographic characteristics of the participants

\begin{tabular}{lc}
\hline & $\mathbf{n}(\mathbf{\%})$ \\
\hline Person completing questionnaire & \\
Mother & $253(75.7)$ \\
Father & $73(21.9)$ \\
Stepmother & 0 \\
Stepfather & 0 \\
Other & $8(2.4)$ \\
Parent age (years) & \\
$<20$ & $3(0.9)$ \\
$20-29$ & $58(17.4)$ \\
$30-39$ & $182(54.5)$ \\
$>40$ & $90(26.9)$ \\
Marital Status & \\
Single & $4(1.2)$ \\
Married & $300(89.8)$ \\
Divorced & $28(8.4)$ \\
Widowed & $1(0.3)$ \\
Nationality & \\
Qatari & $50(15)$ \\
Non-Qatari & $284(85)$ \\
Level of education & \\
Less than high school & $21(6.3)$ \\
High school & $77(23.1)$ \\
Bachelor degree & $182(54.5)$ \\
Higher than Bachelor & $54(16.2)$ \\
Employment status & \\
Not working & $131(39.2)$ \\
Working part time & $26(7.8)$ \\
Working full time & $166(49.7)$ \\
Student (full time) & $3(0.9)$ \\
Student (part time) & $2(0.6)$ \\
Studying and working & $6(1.8)$ \\
Number of children & $40(12)$ \\
2 & $39(11.7)$ \\
\hline & \\
\hline & \\
\hline &
\end{tabular}

media. In terms of preferred age to receive the vaccine, $45 \%$ of the participants preferred to administer the vaccine to their children before they are mature enough to understand sexual relations, while $22 \%$ recommended vaccination right before marriage and $15 \%$ preferred to wait till they are grown up and decide for themselves. Furthermore, only $42 \%$ of the caregivers agreed that it is important to explain to their children the vaccine can protect against some sexually-transmitted infections. More details regarding questions related to HPV vaccine acceptability are displayed in Table 2.

Approximately $20 \%$ of the participants were not convinced about the HPV vaccine. When inquired about the reasons, $42 \%$ doubted the safety of the vaccine, while $13 \%$ stated that the vaccine has been developed for marketing goals, $15 \%$ preferred not to talk to children about a vaccine related to sexual relations and the rest mentioned that cancer is a disease for old people. Sources 
Table 2: Questions related to HPV vaccine acceptability

\begin{tabular}{|c|c|c|c|}
\hline Questions & Yes, $n(\%)$ & No, $n(\%)$ & $\begin{array}{l}\text { Do not know/ } \\
\text { remember } n(\%)\end{array}$ \\
\hline Have you ever heard of genital cancer including cervical cancer? & $220(65.9)$ & $99(29.6)$ & $14(4.2)$ \\
\hline Have you ever heard of the human papilloma virus (HPV, a virus that causes genital cancer?) & $113(33.8)$ & $206(61.7)$ & $14(4.2)$ \\
\hline $\begin{array}{l}\text { Do you feel comfortable giving your child a vaccine that would prevent her or him from } \\
\text { getting genital cancer? }\end{array}$ & $260(77.9)$ & $39(11.7)$ & $34(10.2)$ \\
\hline Has your child's doctor ever recommended HPV vaccine? & $13(3.9)$ & $288(86.2)$ & $32(9.6)$ \\
\hline If the HPV vaccine can protect your child from cancer, would you give it to him or her? & $269(80.5)$ & $36(10.8)$ & $28(8.4)$ \\
\hline
\end{tabular}

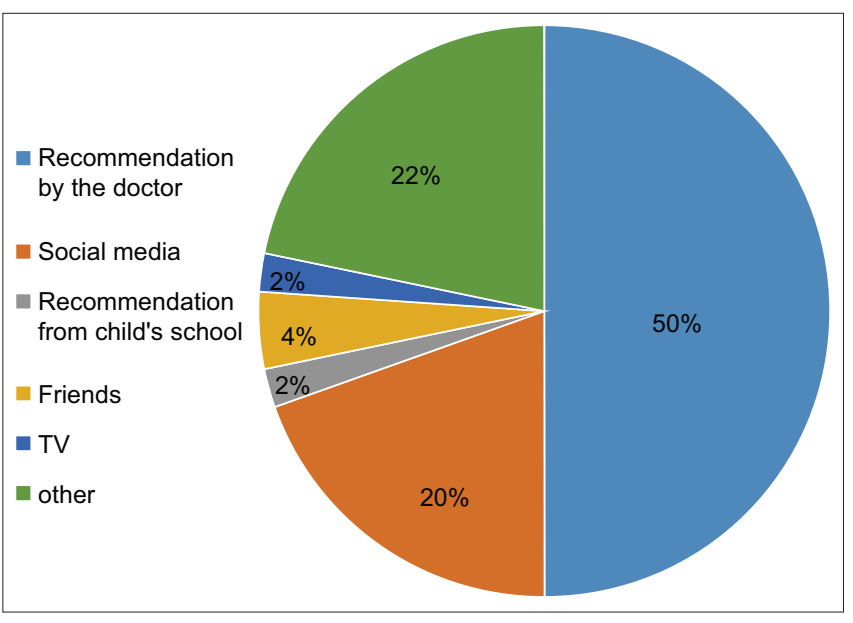

Figure 1: Sources that would positively influence parents to accept the vaccine

that would positively influence parents to accept the vaccine after refusal are shown in Figure 1.

Married caregivers were more open to the idea of giving the HPV vaccine to their children if recommended by the child's primary physician $(P=0.045)$. Being a Qatari national was associated with the preference for receiving information about the HPV vaccine from the clinician's office $(P=0.005)$. The remaining associations among sociodemographic factors and questions related to the acceptability of the HPV vaccine among caregivers were not statistically significant $(P>0.05)$.

\section{Discussion}

The current study is the first study in Qatar to examine parental perceptions toward the HPV vaccine. It measured parental insights and concerns about the general and specific aspects of administering the vaccine to their children. Overall, our participating population was positive and receptive to the idea of the HPV vaccine.

Caregivers play a crucial role in caring for their children and making choices for their beloved ones. ${ }^{[17]}$ Their views and understanding of care may differ from those perceived by clinicians. Delineating parental perceptions is vital to evolving programs and solutions and is essential to the founding of patient-centered care. ${ }^{[18]}$
Several investigations have shown that despite the misconceptions about the topics, HPV and cervical cancer, parents are positive toward HPV vaccination and vaccination against other sexually transmitted infections (STI's). ${ }^{[10-13]}$

In 2018, Gamaoun ${ }^{[14]}$ conducted a systematic review that included 18 studies involving nine Arab states. Qatar was not included in the study. Knowledge and awareness of the HPV infection ranged from 4.2 to $97.0 \%$ depending on the state and subpopulation. It was utmost among the subgroup that included clinicians $(97.0 \%)$ and lowest among the subgroup of parents (varying between 4.2 and $18.0 \%$ ). Knowledge and awareness of the causal link between the HPV infection-cervical cancer was as low as $8.4 \%$ in parents. As for HPV vaccine awareness, the prevalence was found among the parents ranging from 14.2 to $34.2 \%$, depending on the country. Up to $99 \%$ of parents showed acceptability of the vaccine. Our results were not far from the average prevalence in the Arab world.

The high acceptance rate found in this study could be attributed to different factors. First, the citizens and residents in the State of Qatar trust the decrees issued by the ministry of health, and parents are accustomed to having their children vaccinated. Second, for many parents, their participation in this survey was their first contact with a provided well-presented fact sheet. Thirdly, the positive attitude toward HPV vaccination could be elucidated because parents gave the answer they perceived would be the most socially desirable, instead of providing the response they truly wanted to give. However, this desire was minimized by emphasizing that their participation was voluntary and that their answers were anonymous and confidential.

The role of health care professionals can influence the decision to accept or not accept HPV vaccination, therefore, they could play a pivotal role in the HPV vaccine programs. Primary care clinicians have a family-centered perspective and are seen by families as a reliable source of health counseling. ${ }^{[19]}$ This observation was clear in our study and was consistent with the data published by Zimet ${ }^{[20]}$ which showed that the success of HPV vaccine programs depends on the motivation and competency of clinicians to recommend this vaccine to the target population.

Most of the parents conveyed their predilection to vaccinate their children when they are grown-up or set to marry. This could mirror either the conservative social status of the parents to a 
new vaccine or the belief among them that premarital sex is still not common among adolescents residing in Qatar.

It has been assumed that many of the parents, especially in conservative societies, might perceive that sexual health programs and the administration of a vaccine for a sexually acquired virus will incite unsafe sexual relations. ${ }^{[21]}$ However, we sensed that this was not a major issue.

Vaccines, in general, are acceptable in our study population. Despite this, $20 \%$ of our parents were skeptical of the idea of the HPV vaccine. The most common reason for refusal was concern about the safety of the new vaccine. Beliefs in the safety of the vaccine in the study concurred with the published literature. ${ }^{[22]}$

The State of Qatar is a prosperous country and any vaccine program is feasible to be applied.

This study has important strengths, specifically both the quantitative and qualitative observations. Our study will assist in illustrating the parental attitude toward the HPV vaccine in Qatar and will contribute to adding this vaccine to the National Immunization Schedule.

This study also has limitations. There might be a possibility that there are certain aspects related to parental attitude in this subject that were not evaluated in this study. Moreover, this study collected perceptions from parents and delineating the teenagers' perceptions would be invaluable.

\section{Conclusion}

A large proportion of parents residing in Qatar have a positive perception regarding the HPV vaccine. Parents' attitudes and perceptions are considered indispensable targets for community health intervention. We will share the result of our study with the ministry of public health in Qatar with a goal to incorporate the $\mathrm{HPV}$ vaccine in the National Immunization Schedule.

\section{Key points}

* More than $60 \%$ of the parents were not aware that HPV can cause cervical and genital cancer.

* About $80 \%$ of the participants were convinced about the $\mathrm{HPV}$ vaccine.

* Married caregivers were more open to the idea of giving the HPV vaccine to their children if recommended by the child's primary physician.

\section{Table of contents summary}

This study describes parental perspectives and challenges in introducing the HPV vaccine in Qatar.

\section{Acknowledgments}

Open access article processing fees were covered by Qatar National Library.

\section{Ethical approval}

Sidra Medicine IRB approved this study (\#IRB 2019-0003)

\section{Declaration of patient consent}

The authors certify that they have obtained all appropriate patient consent forms. In the form the patient(s) has/have given his/her/their consent for his/her/their images and other clinical information to be reported in the journal. The patients understand that their names and initials will not be published and due efforts will be made to conceal their identity, but anonymity cannot be guaranteed.

\section{Financial support and sponsorship}

The article processing fees are covered by Qatar National Library.

\section{Conflicts of interest}

There are no conflicts of interest.

\section{References}

1. Martel C, Georges D, Bray F, Ferlay J, Clifford GM. Global burden of cancer attributable to infections in 2018: A worldwide incidence analysis. Lancet Glob Health 2020;8:e180-90. doi: 10.1016/S2214-109X (19) 30488-7.

2. Cervical Cancer. World Health Organization. Available from https://www.who.int/health-topics/cervicalcancer\#tab=tab_1 [Last accessed 01 Jul 2021].

3. Nations online. Qatar. Available from: https://www. nationsonline.org/oneworld/qatar.htm. [Last accessed on 2020 May 24].

4. Human Papillomavirus and Related Diseases Report. Qatar. Available from: http://www. hpvcentre.net/statistics/ reports/QAT.pdf. [Last accessed on 01 Jul 2021].

5. Basu P, Mittal S. Acceptability of human papillomavirus vaccine among the urban, affluent and educated parents of young girls residing in Kolkata, Eastern India. J Obstet Gynaecol Res 2011;37:393-401.

6. The Center for Disease Control and Prevention (CDC). Human papilloma virus. Genital HPV Infection-Fact Sheet. Available from: https://www.cdc.gov/std/hpv/stdfact-hpv. htm. [Last accessed on 2020 May 28].

7. Kjaer SK, Nygård M, Sundström K, Dillner J, Tryggvadottir L, Munk C, et al. Final analysis of a 14-year long-term follow-up study of the effectiveness and immunogenicity of the quadrivalent human papillomavirus vaccine in women from four nordic countries. EClinicalMedicine 2020;23:100401.

8. Brisson M, Kim JJ, Canfell K, Drolet M, Gingras G, Burger EA, et al. Impact of HPV vaccination and cervical screening on cervical cancer elimination: A comparative modelling analysis in 78 low-income and lower-middle-income countries. Lancet 2020;395:575-90.

9. Agosti JM, Goldie SJ. Introducing HPV vaccine in developing countries - Key challenges and issues. N Engl J Med 2007;356:1908-10.

10. Ngcobo NJ, Burnett RJ, Cooper S, Wiysonge CS. Human papillomavirus vaccination acceptance and hesitancy in South Africa: Research and policy agenda. S Afr Med J 2018;109:13-5. 
11. DiAnna Kinder F. Parental refusal of the human papillomavirus vaccine. J Pediatr Health Care 2016;30:551-7.

12. Spagnoletti BR, Bennett LR, Wahdi AE, Wilopo SA, Keenan CA. A qualitative study of parental knowledge and perceptions of human papillomavirus and cervical cancer prevention in Rural Central Java, Indonesia: Understanding community readiness for prevention interventions. Asian Pac J Cancer Prev 2019;20:2429-34.

13. Victory M, Do TQN, Kuo YF, Rodriguez AM. Parental knowledge gaps and barriers for children receiving human papillomavirus vaccine in the Rio Grande Valley of Texas. Hum Vaccin Immunother 2019;15:1678-87.

14. Gamaoun R. Knowledge, awareness and acceptability of anti-HPV vaccine in the Arab states of the Middle East and North Africa region: A systematic review. East Mediterr Health J 2018;24:538-48.

15. Jaspers L, Budiningsih S, Wolterbeek R, Henderson FC, Peters AA. Parental acceptance of human papillomavirus (HPV) vaccination in Indonesia: A cross-sectional study. Vaccine 2011;29:7785-93.

16. Zhang SK, Pan XF, Wang SM, Yang CX, Gao XH, Wang ZZ, et al. Perceptions and acceptability of HPV vaccination among parents of young adolescents: A multicenter national survey in China. Vaccine 2013;31:3244-9.

17. Sobo EJ, Kurtin P. Variation in physicians' definitions of the competent parent and other barriers to guideline adherence: The case of pediatric minor head injury management. Soc Sci Med 2003;56:2479-91.

18. Sobo EJ, Seid M, Reyes Gelhard L. Parent-identified barriers to pediatric health care: A process-oriented model. Health Serv Res 2006;41:148-72.

19. Kokorelias KM, Gignac MA, Naglie G, Cameron JI. Toward a universal model of family centered care: A scoping review. BMC Health Serv Res 2019;19:564.

20. Zimet GD. Improving adolescent health: Focus on HPV vaccine acceptance. J Adolesc Health 2005;37 (6 Suppl):S17-23.

21. Brewer NT, Fazekas KI. Predictors of HPV vaccine acceptability: A theory-informed, systematic review. Prev Med 2007;45:107-14.

22. Brabin L, Roberts SA, Farzaneh F, Kitchener HC. Future acceptance of adolescent human papillomavirus vaccination: A survey of parental attitudes. Vaccine 2006;24:3087-94. 